Quim. Nova, Vol. 36, No. 9, 1308-1317, 2013

\title{
DESCRIPTORES GLOBALES Y LOCALES DE LA REACTIVIDAD PARA EL DISEÑO DE NUEVOS FÁRMACOS ANTICANCEROSOS BASADOS EN CIS-PLATINO(II)
}

\author{
Jesús M. López*, Adolfo E. Ensuncho y Juana R. Robles \\ Departamento de Química, Universidad de Córdoba, Grupo de Química Computacional, Cra. 6 N 74-103, Córdoba-Colombia
}

Recebido em 6/2/13; aceito em 21/6/13; publicado na web em 2/8/13

\begin{abstract}
GLOBAL AND LOCAL REACTIVITY DESCRIPTORS FOR THE DESIGN OF NEW ANTICANCER DRUGS BASED ON CISPLATINUM(II). Density functional theory was used to investigate the global and local reactivity of some cis-platinum(II) complexes including anticancer drugs, such as cisplatin and carboplatin. Calculated equilibrium geometries at mPW1PW/LANL2DZ* are in close agreement with their available X-ray data. We develop three new local reactivity descriptors: atomic descriptor of philicity, atomic descriptor group and atomic descriptor of philicity group for determining chemical reactivity and selectivity of the studied complexes. This contribution on chemical reactivity allow us to establish qualitative trends, which enable our descriptors for use in rational platinum based anticancer drug design.
\end{abstract}

Keyword: DFT calculations; cisplatin; electrophilicity index.

\section{INTRODUCCIÓN}

Las novedosas técnicas de modelización y las aproximaciones desarrolladas en el campo de la teoría de estructura electrónica molecular, están asumiendo un papel importante en las investigaciones de química cuántica medicinal y en el diseño de nuevos agentes terapéuticos para el tratamiento de numerosas patologías. ${ }^{1-5}$ Dentro de los temas de investigación en el área de la química cuántica medicinal, un campo interesante de estudio comprende la química del fármaco anticanceroso cisplatino (cis-diaminodicloroplatino(II)), el cual desde su descubrimiento hace más de cuatro décadas por el biofísico Rosenberg, ${ }^{6}$ se ha convertido en un paradigma para el tratamiento de tumores testiculares y ováricos. ${ }^{7}$ Este notable interés por investigar la estructura electrónica del fármaco anticanceroso cisplatino, se debe al hecho que, aun cuando existe información experimental suficiente sobre los principales aspectos farmacodinámicos que gobiernan la acción antitumoral de este agente, se requiere el desarrollo de modelos teóricos que permitan dar soporte a la evidencia experimental generada, pues aun existen muchos interrogantes con respecto al modo de acción de este fármaco que todavía no han sido totalmente comprendidos, ${ }^{5}$ pues si bien es cierto, que el mecanismo de acción tradicionalmente establecido para cisplatino indica que éste, se une al ADN formando aductos inter e intrahebra de los cuales los más abundantes son aquellos donde se forma un enlace 1,2-intrahebra entre los átomos de $\mathrm{N}(7)$, de dos bases guanina adyacentes, ${ }^{8}$ aun permanecen aspectos sin desentrañar (material Suplementario, Figura 1S). ${ }^{9}$ De modo que en su aspecto más simple, la unión Pt-N(7), corresponde a una interacción electrófilo/nucleófilo.

Por otro lado, aunque cisplatino es un fármaco anticanceroso exitoso, (con tasa de curación que supera el 90\%), presenta una serie de reacciones colaterales indeseables que limitan su aplicación terapéutica. ${ }^{10}$ Además, se han encontrado casos de resistencia de algunos tumores frente a la acción del fármaco. ${ }^{11}$ En razón a lo anterior, se han sintetizado números compuestos análogos a cisplatino con el fin de superar estos inconvenientes; por lo que en la actualidad los análogos carboplatino y oxaliplatino recibieron su aprobación por la Administración de Alimentos y Medicamentos (FDA, por sus siglas en inglés) y se usan en regímenes quimioterapéuticos rutinarios. ${ }^{12}$

*e-mail: jmanuellopez.gqc@gmail.com
Asimismo, en países como Japón, China y Corea, los fármacos, nedaplatino, lobaplatino y SKI2053R, respectivamente se encuentran bajo uso, pero con restricciones. ${ }^{13}$ No obstante, aunque estos nuevos derivados, presentan ciertas ventajas sobre el fármaco de referencia todavía existen algunos inconvenientes. Por lo tanto, la búsqueda de mejores agentes anticancerosos basados en platino continua, atendiendo ciertos requerimientos tales como: elevada actividad antitumoral, menor toxicidad hacia las células sanas y que eviten la resistencia celular. ${ }^{14} \mathrm{~A}$ este propósito, tanto experimental, así como también, teóricamente se desarrollan estrategias para el diseño racional de nuevos fármacos anticancerosos basados en platino(II), empleando las herramientas computacionales para caracterizar en principio los sistemas de interés y con ello asistir las síntesis de los potenciales agentes antitumorales. Así por ejemplo, en la literatura científica, de las aproximaciones computacionales disponibles, la Teoría del Funcional de la Densidad (DFT, por sus siglas en inglés), ${ }^{15}$ ha mostrado ser un método clave para el diseño de fármacos y específicamente en el campo de los metales de transición con aplicación medicinal, se han obtenido resultados satisfactorios en cuanto a precisión y confiabilidad. ${ }^{5} \mathrm{El}$ aspecto conceptual de esta teoría, se ha utilizado notablemente para comprender la reactividad química de los sistemas químicos, ${ }^{16,17}$ pues de las derivadas de la DFT, se ha logrado definir en forma rigurosa conceptos tales como la electronegatividad y la dureza, los cuales son importantes índices de reactividad. El potencial químico electrónico $(\mu)$, la dureza $(\eta)$, la electrofilicidad $(W)$ y la blandura (S), respectivamente, corresponden a descriptores globales de la reactividad, ${ }^{18}$ mientras que la función de Fukui (FF), la filicidad $\left(\omega_{k}^{\alpha}\right)$ y la suavidad local $\left(s_{k}^{\alpha}\right)$, son descriptores locales de la reactividad. ${ }^{18-20}$ Este conjunto de descriptores se han usado con éxito, para entender la toxicidad y actividad biológica de moléculas orgánicas, ${ }^{19,21}$ sin embargo, para compuestos inorgánicos tales como cisplatino y derivados hay pocos estudios que muestren la importancia de estos descriptores mecanocuánticos para entender la naturaleza reactiva de estas moléculas y la estrecha relación que existe entre la estructura y su actividad farmacológica. ${ }^{22,23}$ De lo anterior, es claro que se hace necesario desarrollar estudios sistemáticos enfocados en la caracterización de la reactividad de moléculas análogas a cisplatino, con el fin de predecir tendencias cualitativas de reactividad para complejos derivados que aun no han sido sintetizados ni probados en cuanto a su actividad farmacológica y que además permitan idear protocolos 
de diseño para futuros prospectos con potencialidad anticancerosa. Por ello, en el presente trabajo de calculan el conjunto de descriptores globales y locales de la reactividad previamente mencionados, para una serie de análogos de cisplatino recientemente sintetizados por Moradell y colabores ${ }^{24,25}$ y Dalla Via y colaboradores, ${ }^{10} \mathrm{e}$ incluyendo los fármacos de uso clínico actual cisplatino y carboplatino. Esto se llevo a cabo con el propósito de establecer la importancia de estas cantidades en el análisis de las características reactivas de este grupo de moléculas y su utilidad para diseñar nuevos complejos análogos que aun no han sido reportados en literatura. El grupo de complejos seleccionados para los cálculos DFT, hacen parte de las nuevas aproximaciones usadas para el desarrollo de nuevos agentes anticancerosos basados en platino(II), la cual consiste en coordinar al centro metálico ligandos fisiológicamente activos, ya que con esta estrategia se han obtenido resultados interesantes en cuanto a actividad y especificidad. ${ }^{26,27}$ Para el caso, los ligandos bioactivos coordinados al átomo de platino corresponden a los aminoácidos alanina, fenilalaina y ornitina respectivamente. Además, se propusieron 10 complejos análogos a cisplatino que aun no han sido sintetizados con base en las reglas empíricas de Cleare y Hoeschele, ${ }^{28}$ bajo las cuales para que un fármaco anticanceroso basado en platino presente actividad debe tener como prerrequisito necesario que dos ligandos amino bidentados o aminos se encuentren en conformación cis con dos ligandos de fuerza de enlace intermedia, como por ejemplo, cloruros, sulfatos, citrato u oxalato con respecto al centro metálico. ${ }^{29}$ Los ligandos amino, suelen denominarse ligandos portadores (LP), mientras que los grupos cloruro y oxalato son designados como ligandos salientes (LS). ${ }^{28}$ En este orden de ideas, para los 10 análogos propuestos se varió tanto la naturaleza de los grupos portadores como la de los grupos salientes. Así por ejemplo, como ligandos portadores fueron seleccionados los aminoácidos esenciales; valina, triptófano, leucina, tirosina e hístidina respectivamente y los ligandos salientes; 1,1'-ciclobutanodicarboxilato y cloruro respectivamente, ya que, es un hecho experimental conocido que la citotoxicidad de cisplatino y análogos, es modulada por estos ligandos..$^{30}$ Además, para el análisis de las tendencias de reactividad de los sistemas modelo y los 10 análogos propuestos, se definieron tres nuevos descriptores para describir la reactividad y selectividad de sitio de estas moléculas (descriptores locales), basados en los descriptores de filicidad de grupo $\left(\omega_{g}^{\alpha}\right),{ }^{20}$ suavidad de grupo $\left(s_{g}^{\alpha}\right)^{31}$ y el descriptor atómico $s_{k}^{\alpha} f_{k}^{\alpha}$, recientemente introducido por Kolandaivel y colaboradores, ${ }^{32}$ con los cuales se espera obtener información valiosa sobre diferentes sitios de las moléculas investigadas.

\section{FUNDAMENTOS TEÓRICOS}

La teoría del funcional de la densidad (DFT), se ha utilizado para entender la reactividad química y la selectividad de sitio de los sistemas moleculares. ${ }^{16-18,31,33-47}$ Las ecuaciones básicas de la DFT, se obtienen a través de la minimización del funcional de energía de la densidad electrónica, $\mathrm{E}[\rho]$ con la restricción que el número de electrones $(\mathrm{N})$ permanezca constante, conduciendo a la expresión mostrada en la Ecuación 1:18

$$
\delta\left(E-\mu \int \rho(r) d r\right)
$$

donde, $\int \rho(r) d r=N$ y $\mu$ es el multiplicador de Lagrange, el cual corresponde al potencial químico electrónico ${ }^{38}$ y que además está relacionado con el concepto de electronegatividad de Pauling $(\chi=-\mu) .^{48}$ Resolviendo variacionalmente la Ecuación 2, se alcanza la ecuación de Euler-Lagrange a saber: ${ }^{38}$

$$
\mu=v(r)+\frac{\delta F[\rho(r)]}{\delta \rho(r)}
$$

donde, $v(r)$, es el potencial externo y $F[\rho(r)]$, es el funcional universal, que contiene la energía cinética, la energía de repulsión electrón-electrón (Hartree-Coulomb) y la energía de correlación e intercambio respectivamente, de acuerdo con los teoremas de Hohenberg y Kohn. ${ }^{49}$ El potencial químico $(\mu)$ y la dureza $(\eta)$ pueden definirse como la primera y segunda derivada de la energía E con respecto al número total de electrones a potencial externo $v(r)$ constante: ${ }^{38}$

$$
\mu=\left(\frac{\partial E}{\partial N}\right)_{v(r)} ; \eta=\frac{1}{2}\left(\frac{\partial^{2} E}{\partial N^{2}}\right)_{v(r)}
$$

Físicamente, el potencial químico electrónico, corresponde a la capacidad de un sistema para donar electrones, de tal modo que la transferencia de electrones, entre reactantes fluye desde el potencial más alto hacia el más bajo, mientras que la dureza puede ser entendida como la resistencia a la transferencia de carga del sistema. ${ }^{50}$ Para efectos prácticos, en muchas aplicaciones numéricas el potencial químico electrónico y la dureza se calculan mediante la energía de ionización (EI) y la afinidad electrónica (AE) respectivamente como se muestra en la Ecuación 4. ${ }^{39,51}$

$$
\begin{aligned}
& \mu=-\frac{1}{2}(E I+A E) \\
& \eta=-\frac{1}{2}(E I-A E)
\end{aligned}
$$

Por otro lado, las expresiones de la Ecuaciones 4a y 4b, se pueden calcular en términos de las energías orbitales basadas en la aproximación de diferencias finitas de tres puntos y el teorema de Koopmans: ${ }^{.5}$

$$
\begin{gathered}
\mu=\frac{1}{2}\left(\varepsilon_{\text {LUMO }}+\varepsilon_{\text {HOMO }}\right) \\
\eta=\frac{1}{2}\left(\varepsilon_{\text {LUMO }}-\varepsilon_{\text {HOMO }}\right)
\end{gathered}
$$

Donde, $\varepsilon_{\text {LUMO }}$ у $\varepsilon_{\text {номO }}$, son las energías de los orbitales moleculares (HOMO y LUMO, por sus siglas en inglés) más bajo desocupado y mas alto ocupado respectivamente. La suavidad ( $\mathrm{S}$ ), es el concepto inverso de la dureza y constituye un concepto útil para la predicción de la reactividad química. Las moléculas blandas sufren cambios en su densidad electrónica más fácilmente que las moléculas duras y por ende son más reactivas. ${ }^{52}$ En general, se puede decir que el incremento en la suavidad se asocia con el incremento de la reactividad química mientras que la dureza está relacionada con la disminución de la reactividad química. Se define como:

$$
S=\frac{1}{2 \eta}
$$

El concepto de electrofilicidad global $(W)$, fue introducido por Parr y colaboradores, ${ }^{53}$ para medir la estabilización de la energía cuando el sistema adquiere carga electrónica adicional proveniente de los alrededores. Se calcula mediante la Ecuación (7).

$$
W=\frac{\mu^{2}}{2 \eta}
$$

Los descriptores $\mu, \eta, W$ y $S$ muestran la reactividad de las moléculas como un todo, sin embargo, para estudiar la reactividad y selectividad de sitio es necesario el uso de descriptores locales de la reactividad. ${ }^{38}$

Los descriptores locales como la función de Fukui (FF), la blandura local $(s(\mathrm{r}))$ y la filicidad $\left(\omega_{k}^{+}\right)$son propiedades que explican la selectividad en una región de una molécula. La FF, se define como: ${ }^{54,55}$ 


$$
f(r)=\left(\frac{\partial \rho(r)}{\partial N}\right)_{v(r)}
$$

La Ecuación (8), presenta un problema de discontinuidad en átomos y moléculas cuando se combina con la aproximación de diferencias finitas dando lugar a tres aproximaciones de la FF como son: ${ }^{51}$

$$
\begin{gathered}
f^{+}(r)=\left(\frac{\partial \rho(r)}{\partial N}\right)_{v(r)}^{+} \\
f_{k}^{-}=\left[q_{k}(N)-q_{k}(N-1)\right] \\
f^{0}(r)=\frac{1}{2}\left[f^{+}(r)+f^{-}(r)\right]
\end{gathered}
$$

De modo que en un punto $r$ del espacio, $f^{+}(r)$, mide la reactividad de un ataque nucleofílico, $f^{-}(r)$ mide la reactividad para un ataque electrofílico y $f^{0}(r)$ describe un ataque por un radical y se obtiene como el promedio aritmético de FF para el ataque nucleofílico y electrofílico respectivamente. Para efectos prácticos, la cuantificación de la FF es posible, a través de un esquema de condensación sobre una región atómica de la molécula, empleando procedimientos de análisis de población, dando lugar a las siguientes Ecuaciones:44,56

$$
\begin{gathered}
f_{k}^{+}=\left[q_{k}(N+1)-q_{k}(N)\right] \\
f_{k}^{-}=\left[q_{k}(N)-q_{k}(N-1)\right] \\
f_{k}^{0}=\frac{1}{2}\left[q_{k}(N+1)-q_{k}(N-1)\right]
\end{gathered}
$$

donde, $q_{k}(N+1), q_{k}(N-1)$ y $q_{k}(N)$ denotan la población electrónica del átomo $k$, en la especie aniónica, catiónica y neutra respectivamente. En esta investigación, se utilizará únicamente la ecuación que describe el ataque nucleofílico (10a), debido a la naturaleza de la interacción entre los complejos de cis-platino(II) con ADN. En analogía a la función condensada de Fukui para el ataque nucleofílico, y la dureza global, para un ataque nucleofílico la suavidad local $\left(S_{k}^{+}\right)$viene dada por: ${ }^{40}$

$$
s_{k}^{+}=f_{k}^{+} S
$$

Donde, $\mathrm{S}$ es la suavidad global y $f_{k}^{+}$es la función de Fukui como se definió en la Ecuación 10a. Además, se puede calcular el índice de electrofilicidad local (filicidad), a partir de una expresión análoga a la Ecuación 11, quedando de la forma: ${ }^{20}$

$$
\omega_{k}^{+}=f_{k}^{+} W
$$

Donde $W$, es la electrofilicidad global y $f_{k}^{+}$es la función de Fukui como se definió en la Ecuación 10a. Este concepto fue introducido por Chattaraj, ${ }^{20}$ para analizar las reacciones entre electrófilos y nucleófilos, por lo que predice que el sitio más electrofílico en una molécula corresponde a aquel con el máximo valor de $\omega_{k}^{+}$. Por otro lado, se sabe que la reactividad intra e intermolecular se puede explicar en términos de $f(r)$ o $s(r),{ }^{57}$ por lo que Kolandaivel y colaboradores ${ }^{32}$ recientemente conectaron $f(r)$ y $s(r)$, en un nuevo descriptor denominado "descriptor atómico" (DA) y definido como $(s f)_{k}^{\alpha}$, el cual también se puede condensar a un sitio atómico de la forma general: ${ }^{32}$

$$
(s f)_{k}^{\alpha}=\left(f_{k}^{\alpha}\right)^{2} S
$$

donde, $\alpha=+/-/ 0$, se refieren al ataque nucleofílico, electrofílico y radical respectivamente. Por analogía a este descriptor atómico, en este trabajo se propone un nuevo descriptor al cual hemos denominado "descriptor atómico de filicidad", asociado con la condición de normalización de la función de Fukui ${ }^{20}$ y definido de la forma (Material Suplementario):

$$
(\omega f)_{k}^{\alpha}=\left(f_{k}^{\alpha}\right)^{2} W
$$

donde, $\alpha=+/-/ 0$, se refieren al ataque nucleofílico, electrofílico y radical respectivamente. Con este nuevo parámetro, se espera obtener información útil de la reactividad de los sitios locales durante el ataque nucleofílico de ADN sobre los complejos de cis-platino(II) investigados. Otros descriptores locales de significativa importancia, corresponden a los definidos dentro del contexto de la aproximación de grupo, como lo son la filicidad y suavidad de grupo. Se definen a través de las Ecuaciones siguientes: ${ }^{31,58}$

$$
\begin{gathered}
S_{g}=\sum_{k=1}^{n} s_{k} \\
\omega_{g}^{\alpha}=\sum_{k=1}^{n} \omega_{k}^{\alpha}
\end{gathered}
$$

De la Ecuación 15a, n, corresponde al número de átomos vecinos al centro de reacción, $\omega_{k}^{\alpha}$ es la filicidad, los superíndices $\alpha=+/-/ 0$, se refieren al ataque nucleofílico, electrofílico y radical respectivamente. La utilidad de estos descriptores, para predecir tendencias de reactividad de sistemas químicos ya ha sido demostrada. ${ }^{31}$ Por lo tanto, en la exploración de nuevos descriptores para obtener información de la reactividad de los sistemas químicos, en este trabajo, se propone la definición del "descriptor atómico de filicidad de grupo" y "descriptor atómico de grupo" respectivamente, definidos mediante las ecuaciones:

$$
\begin{gathered}
(s f)_{g}^{\alpha}=\sum_{k=1}^{n}(s f)_{k}^{\alpha} \\
(\omega f)_{g}^{\alpha}=\sum_{k=1}^{n}(\omega f)_{k}^{\alpha}
\end{gathered}
$$

Los nuevos descriptores de grupo presentados aquí, se definen para el ataque nucleofílico, electrofílico y radical respectivamente $(\alpha=+/-/ 0)$. En este trabajo, se analizará la utilidad de estos descriptores basados en la aproximación de grupo para predecir la reactividad intermolecular de una serie de complejos de cis-platino(II), con actividad anticancerosa establecida, así como también, de análogos que aun no han sido sintetizados ni ensayados.

\section{METODOLOGÍA}

Todos los cálculos computacionales se llevaron a cabo con el paquete de programas Gaussian 09 (Rev B.01). ${ }^{59}$ Los complejos de cis-platino(II), se optimizaron completamente sin restricciones a la geometría, usando los funcionales $\mathrm{B} 3 \mathrm{LYP},{ }^{60} \mathrm{mPW} 1 \mathrm{PW}$, introducido por Adamo y Barone ${ }^{61}$ y el funcional introducido por Hamprecht y colaboradores HCTH. ${ }^{62}$ Las estructuras optimizadas se caracterizaron como mínimos, mediante cálculos de frecuencias vibracionales armónicas. ${ }^{63}$ El potencial químico electrónico $(\mu)$, la dureza $(\eta)$, el índice de electrofilicidad $(W)$ y blandura $(\mathrm{S})$, se calcularon mediante las Ecuaciones 5a, 5b, 6 y 7 respectivamente. La función de Fukui, se calculó mediante el análisis de población de Hirshfeld (HPA), ${ }^{64}$ implementado en el programa Gaussian 09. Varios trabajos de investigación han reportado la utilidad de este esquema de análisis de población, para la determinación de descriptores locales..$^{20,65} \mathrm{La}$ función de Fukui, filicidad y suavidad local para el ataque nucleofílico, 
se calcularon a partir de las Ecuaciones 10a, 11 y 12 respectivamente. Los descriptores atómicos $(s f)_{k}^{\alpha}$ y $(\omega f)_{k}^{\alpha}$ se calcularon mediante las Ecuaciones 13 y 14 respectivamente, mientras que los nuevos descriptores definidos mediante la aproximación de grupo, y que de ahora en adelante designaremos como DG, se calcularon empleando las Ecuaciones 16a y $16 \mathrm{~b}$ respectivamente. Las geometrías optimizadas en fase gas de los diferentes complejos de cis-platino(II) investigadas, se reoptimizaron en fase acuosa usando el modelo de solvente del continuo polarizado $\mathrm{PCM}^{66}$ al mismo nivel de teoría. Se usó una constante dieléctrica de 78,5 a una temperatura de $25^{\circ} \mathrm{C}$, correspondiente a la constante dieléctrica experimental del agua a dicha temperatura. ${ }^{67}$ Asimismo, se usó el potencial efectivo del core LANL2DZ de Hay y Wadt ${ }^{68}$ para reemplazar los 60 electrones de la capa más interna del átomo de platino y para los 18 electrones de valencia restantes se incluirán implícitamente en el cálculo usando la base doble $\zeta$, asociada a LANL2DZ. ${ }^{69} \mathrm{~A}$ su vez, la base LANL2DZ se aumentó con una función de polarización d, con un exponente $\alpha$ de 0,0747 para el átomo de platino, ${ }^{70}$ la cual representaremos de ahora en adelante como LANL2DZ*. Para el resto de átomos, como lo son; cloro, carbono, nitrógeno, oxígeno e hidrógeno se usó la base doble $\zeta$ polarizada $6-31 \mathrm{G}(\mathrm{d}){ }^{63}$

\section{RESULTADOS Y DISCUSIÓN}

Las geometrías de los complejos de cis-platino(II) considerados como sistemas modelo para el estudio computacional, se presentan en la Figura 1a. La nomenclatura adoptada para este grupo de moléculas, correspondió a la asignada por Moradell y colaboradores ${ }^{24,25}$ y Dalla Via y colaboradores, ${ }^{10}$ respectivamente. Para los nuevos derivados propuestos, las geometrías y su respectiva nomenclatura se presentan en la Figura 1b y Tabla 1. La numeración sobre los complejos 2, 10 y VI, indica el grupo de átomos seleccionados para el análisis estructural.

Como se observa en la Figura 1b, para los complejos propuestos se modificaron los grupos salientes y portadores. Así por ejemplo, los ligandos cloruro y $1,1^{\prime}$-ciclobutanodicarboxilato corresponden a grupos salientes, mientras que los grupos portadores se modificaron cambiando la naturaleza del aminoácido quelado al centro de platino(II). Los aminoácidos elegidos fueron; triptófano, valina, leucina, tirosina e hístidina respectivamente.

En la Tabla 2, se presentan los parámetros geométricos predichos en fase gas y acuosa para los complejos estudiados. Evidentemente, de la Tabla 2, se observa que el funcional híbrido de un parámetro mPW1PW, permitió hacer una descripción adecuada de las geometrías de equilibrio para el grupo de moléculas estudiadas, pues, los parámetros geométricos fueron muy cercanos a los datos experimentales. Comparado con los funcionales B3LYP y HCTH, el funcional mPW1PW, fue adecuado para la localización de las geometrías de equilibrio. Además, es de nuestro conocimiento que este funcional ya había sido usado previamente por Wysokinski y Michalska,${ }^{70}$ quienes indicaron que el protocolo mPW1PW/LANL2DZ, es claramente superior a otros funcionales (incluyendo el popular B3LYP), para determinar la geometría de cisplatino y carboplatino respectivamente. De igual manera, el protocolo mPW1PW/LANL2DZ*, también fue adecuado para la descripción de los parámetros geométricos del complejo $\mathrm{PtCl}_{2}[(\mathrm{~L})-\mathrm{N}, \mathrm{O}$-ornitina], cuyos cálculos computacionales son presentados por primera vez. En términos generales, la geometría predicha por este funcional para $\mathrm{PtCl}_{2}[(\mathrm{~L})-\mathrm{N}, \mathrm{O}$-ornitina], mostró concordancia con los datos de difracción de rayos $\mathrm{X}$ reportados por Dalla Via y colaborares para este complejo. ${ }^{10}$ En todos los casos, los complejos mantuvieron una geometría cuadrado-planar con ángulos próximos a los valores ideales de $90^{\circ}$ y $180^{\circ}$ respectivamente. ${ }^{71}$ Por otro lado, como se observa en la Tabla 2, la inclusión del solvente permitió mejorar la descripción de los parámetros geométricos para a)

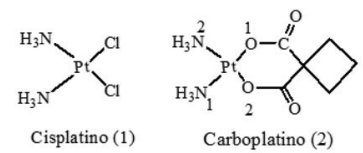<smiles>O=C(O)C1NCP(Cl)(Cl)(Cl)N1</smiles>

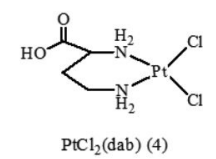<smiles>CC(C)NC(=O)C1CNP(Cl)(Cl)(Cl)N1</smiles><smiles>COC(C)NC(=O)C1CCNP(Cl)(Cl)=N1</smiles>

$\mathrm{PtCl}_{2}$ (dap-ala) (5) $\mathrm{PtCl}_{2}$ (dab-ala) (6)

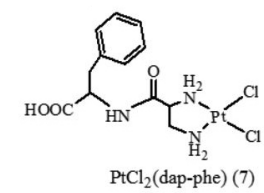

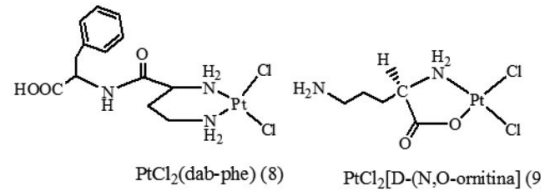

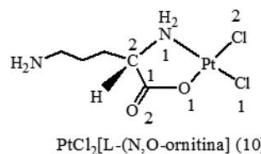

b)<smiles>CC(C)[C@H]1N[PH](Cl)(Cl)OC1=O</smiles>

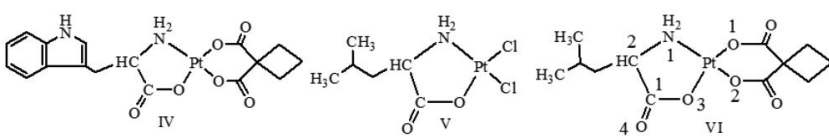

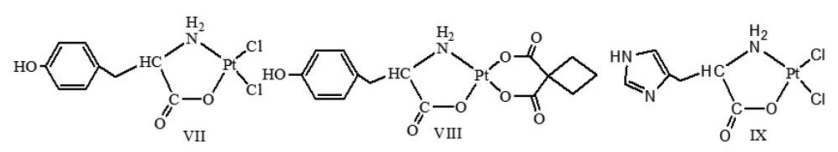

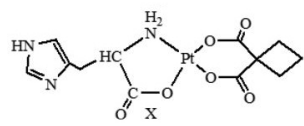

Figura 1. Estructuras de los complejos investigados. 1a) sistemas modelo; 1b) nuevos derivados propuestos

Tabla 1. Nomenclatura para los complejos análogos propuestos

\begin{tabular}{lc}
\hline Complejo & Etiqueta \\
\hline cis-dicloro[N,O-valina]platino(II) & $\mathrm{I}$ \\
cis-(1,1'-ciclobutanodicarboxilato)-[N,O-valina]platino(II) & $\mathrm{II}$ \\
cis-dicloro[N,O-Triptófano]platino(II) & $\mathrm{III}$ \\
cis-(1,1'-ciclobutanodicarboxilato)-[N,O-Triptófano]platino(II) & $\mathrm{IV}$ \\
cis-dicloro[N,O-Leucina]platino(II) & $\mathrm{V}$ \\
cis-(1,1'-ciclobutanodicarboxilato)-[N,O-Leucina]platino(II) & $\mathrm{VI}$ \\
cis-dicloro[N,O-Tirosina]platino(II) & $\mathrm{VII}$ \\
cis-(1,1'-ciclobutanodicarboxilato)-[N,O-Tirosina]platino(II) & $\mathrm{VIII}$ \\
cis-dicloro[N,O-Histidina]platino(II) & $\mathrm{IX}$ \\
cis-(1,1'-ciclobutanodicarboxilato)[N,O-Hístidina]platino(II) & $\mathrm{X}$ \\
\hline
\end{tabular}

los complejos bajo los tres funcionales usados. No obstante, con el funcional mPW1PW las distancias de enlace $r(\mathrm{Pt}-\mathrm{Cl})$ y $r(\mathrm{Pt}-\mathrm{N})$ en cisplatino, estuvieron en concordancia con los valores experimentales. De igual manera, los ángulos de enlace calculados en fase acuosa para cisplatino, $\angle(\mathrm{Cl}-\mathrm{Pt}-\mathrm{Cl})$ y $\angle(\mathrm{N}-\mathrm{Pt}-\mathrm{N})$ con el funcional $\mathrm{mPW} 1 \mathrm{PW}$, mostraron desviaciones del orden de $1,37^{\circ}$ y $3,59^{\circ}$ con respecto al valor experimental. ${ }^{71}$ Aunque, los parámetros geométricos para los complejos, $\mathrm{PtCl}_{2}$ (dap), $\mathrm{PtCl}_{2}$ (dab), $\mathrm{PtCl}_{2}$ (dap-ala), $\mathrm{PtCl}_{2}$ (dab-ala), $\mathrm{PtCl}_{2}$ (dap-phe) y $\mathrm{PtCl}_{2}$ (dab-phe) no se reportan, las geometrías 
Tabla 2. Parámetros geométricos calculados en fase gas y acuosa (mostrados en paréntesis) para cisplatino, carboplatino y $\mathrm{PtCl}_{2}[\mathrm{~L}-(\mathrm{N}, \mathrm{O})$-Ornitina $]$. Longitudes de enlace en angstrom y ángulos de enlace en grados

\begin{tabular}{|c|c|c|c|c|}
\hline Funcional & B3LYP & mPW1PW & HCTH & Experimental \\
\hline $\begin{array}{l}\text { Parámetro } \\
\text { geométrico }\end{array}$ & & Cisplatino & & Ref. 71 \\
\hline$r(\mathrm{Pt}-\mathrm{N})$ & $2,118(2,078)$ & $2,089(2,055)$ & $2,111(2,073)$ & $2,011 \pm 0,04$ \\
\hline$r(\mathrm{Pt}-\mathrm{Cl})$ & $2,347(2,384)$ & $2,322(2,358)$ & $2,336(2,372)$ & $2,330 \pm 0,01$ \\
\hline$\angle(\mathrm{N}-\mathrm{Pt}-\mathrm{N})$ & $98(91)$ & $98(92)$ & $98(91)$ & $87 \pm 1,5$ \\
\hline$\angle(\mathrm{Cl}-\mathrm{Pt}-\mathrm{Cl})$ & $95,4(93,4)$ & $95,2(93,3)$ & $95,0(93,3)$ & $91,9 \pm 0,3$ \\
\hline \multirow[t]{2}{*}{$\angle(\mathrm{N}-\mathrm{Pt}-\mathrm{Cl})$} & $\begin{array}{c}178,894 \\
(179,182)\end{array}$ & $\begin{array}{c}177,727 \\
(179,588)\end{array}$ & $\begin{array}{c}177,828 \\
(179,177) \\
\end{array}$ & --- \\
\hline & & Carboplatino & & Ref. 22 \\
\hline$r(\mathrm{Pt}-\mathrm{N} 1)$ & $2,11(2,08)$ & $2,09(2,06)$ & $2,12(2,08)$ & 2,01 \\
\hline$r(\mathrm{Pt}-\mathrm{O} 1)$ & $1,99(2,03)$ & $2,08(2,01)$ & $2,01(2,03)$ & 2,02 \\
\hline$\angle(\mathrm{O} 1-\mathrm{Pt}-\mathrm{N} 1)$ & $79,93(87,60)$ & $80,03(87,51)$ & $80,90(87,98)$ & 88,20 \\
\hline$\angle(\mathrm{N} 1-\mathrm{Pt}-\mathrm{N} 2)$ & $103,78(92,95)$ & $103,68(92,91)$ & $102,65(92,32)$ & 93,60 \\
\hline$\angle(\mathrm{O} 1-\mathrm{Pt}-\mathrm{N} 2)$ & $175,58(178,19)$ & $175,45(177,94)$ & $175,68(177,66)$ & 177,90 \\
\hline \multirow[t]{2}{*}{$\angle(\mathrm{O} 1-\mathrm{Pt}-\mathrm{O} 2)$} & $96,12(91,77)$ & $96,26(92,04)$ & $95,21(91,57)$ & 88,90 \\
\hline & \multicolumn{3}{|c|}{$\mathrm{PtCl}_{2}[\mathrm{~L}-(\mathrm{N}, \mathrm{O})$-Ornitina $]$} & Ref. 10 \\
\hline$r(\mathrm{Pt}-\mathrm{Cl} 1)$ & $2,325(2,372)$ & $2,303(2,345)$ & $2,314(2,361)$ & 2,317 \\
\hline$r(\mathrm{Pt}-\mathrm{Cl} 2)$ & $2,299(2,323)$ & $2,276(2,298)$ & $2,285(2,308)$ & 2,292 \\
\hline$r(\mathrm{O} 1-\mathrm{C} 1)$ & $1,387(1,395)$ & $1,376(1,380)$ & $1,382(1,389)$ & 1,280 \\
\hline$r(\mathrm{C} 1-\mathrm{O} 2)$ & $1,197(1,196)$ & $1,194(1,193)$ & $1,200(1,198)$ & 1,260 \\
\hline$r(\mathrm{Pt}-\mathrm{O} 1)$ & $2,193(2,135)$ & $2,154(2,105)$ & $2,213(2,147)$ & 2,036 \\
\hline$r(\mathrm{Pt}-\mathrm{N} 1)$ & $2,113(2,081)$ & $2,084(2,055)$ & $2,104(2,076)$ & 2,060 \\
\hline$r(\mathrm{~N} 1-\mathrm{C} 2)$ & $1,495(1,499)$ & $1,483(1,486)$ & $1,486(1,490)$ & 1,480 \\
\hline$r(\mathrm{C} 1-\mathrm{C} 2)$ & $1,531(1,522)$ & $1,523(1,516)$ & $1,528(1,521)$ & 1,530 \\
\hline$\angle(\mathrm{Cl} 1-\mathrm{Pt}-\mathrm{Cl} 2)$ & $96,1(92,9)$ & $95,7(92,5)$ & $95,5(92,7)$ & 92,4 \\
\hline$\angle(\mathrm{O} 1-\mathrm{Pt}-\mathrm{N} 1)$ & $79,2(79,1)$ & $79,7(79,2)$ & $78,3(78,4)$ & 83,1 \\
\hline$\angle(\mathrm{Pt}-\mathrm{O} 1-\mathrm{C} 1)$ & $112,1(115,5)$ & $113,6(116,7)$ & $113,7(116,6)$ & 114,0 \\
\hline$\angle(\mathrm{Pt}-\mathrm{N} 1-\mathrm{C} 2)$ & $111,4(112,1)$ & $111,1(111,8)$ & $112,6(112,9)$ & 109,4 \\
\hline$\angle$ (Cl1-Pt-N1) & $172,5(174,0)$ & $172,9(174,4)$ & $172,9(174,1)$ & 173,7 \\
\hline$\angle(\mathrm{Cl} 2-\mathrm{Pt}-\mathrm{O} 1)$ & $170,4(171,9)$ & $170,8(172,2)$ & $169,7(171,4)$ & 175,5 \\
\hline$\angle(\mathrm{O} 1-\mathrm{C} 1-\mathrm{C} 2)$ & $110,8(110,9)$ & $110,9(111,3)$ & $111,1(111,2)$ & 120,0 \\
\hline$\angle$ (N1-C2-C1) & $110,0(109,9)$ & $110,7(110,7)$ & $110,9(110,9)$ & 110,0 \\
\hline
\end{tabular}

predichas mostraron parámetros geométricos consecuentes con lo esperado. De igual manera, la geometría del complejo $\mathrm{PtCl}_{2}[\mathrm{D}-$ (N,O)-ornitina], fue muy similar a la predicha para la su análogo. Las diferentes longitudes de enlace calculadas para $\mathrm{PtCl}_{2}[\mathrm{D}-(\mathrm{N}, \mathrm{O})$ ornitina] con el funcional $\mathrm{HCTH}$, fueron sutilmente mejores que las obtenidas con B3LYP, aunque los ángulos de enlace son levemente mejor representados con este último funcional. Este resultado es consistente con lo planteado originalmente por los desarrolladores del funcional $\mathrm{HCTH},{ }^{62}$ donde se encontró que para algunos compuestos de metales de transición, las geometrías predichas con este funcional eran levemente mejores que las calculadas con el funcional B3LYP. Los valores inusualmente elevados para los ángulos de enlace $\angle(\mathrm{N}-\mathrm{Pt}-\mathrm{N})$ y $\angle(\mathrm{Cl}-\mathrm{Pt}-\mathrm{Cl})$, en fase gas, obtenidos para cisplatino, se deben a interacciones intramoleculares del tipo $\mathrm{N}-\mathrm{H} \cdots \mathrm{Cl}$, las cuales tienden a abrir dichos ángulos de enlace. ${ }^{22} \mathrm{Un}$ aspecto de particular importancia física, que proporciona el funcional mPW1PW consiste en la adecuada descripción de las interacciones de largo alcance (interacciones no covalentes), ${ }^{61}$ de ahí que, los ángulos de enlace $\angle(\mathrm{N}-\mathrm{Pt}-\mathrm{N}), \angle(\mathrm{Cl}-\mathrm{Pt}-\mathrm{Cl})$ y $\angle(\mathrm{N}-\mathrm{Pt}-\mathrm{Cl})$, no sean sobreestimados por efectos de la interacción $\mathrm{N}-\mathrm{H} \cdots \mathrm{Cl}$, y por ende presenten valores más próximos a los experimentales que los predichos por B3LYP y HCTH respectivamente. Con respecto a las geometrías obtenidas para los análogos propuestos, estas fueron similares a las predichas para el complejo $\mathrm{PtCl}_{2}[(\mathrm{~L})-\mathrm{N}, \mathrm{O}-$ ornitina]. En la Tabla 3, se presentan los parámetros geométricos calculados para los complejos I y VI en ambas fases. Se observa para I y VI, que el entorno cuadrado-planar alrededor de platino se mantiene y la coordinación del grupo 1,1'-ciclobutanodicarboxilato, no induce fuertes cambios en la geometría del complejo derivado.

Una realización importante en este trabajo, fue la caracterización teórica de la reactividad química de los complejos investigados, mediante descriptores globales y locales de la reactividad los cuales se encuentran justificados en la DFT conceptual. En la Tabla 4, se presentan los valores de dichos descriptores calculados al nivel mPW1PW/LANL2DZ* para los sistemas modelo (Figura 1a). Antes de iniciar con el análisis de las cantidades globales y locales predichas para el grupo de moléculas estudiadas, es preciso contar con un referente de la reactividad observada experimentalmente para la serie de complejos investigados. Por ello, en la Tabla 5 se presentan los datos de actividad biológica para estas moléculas, y de los cuales se puede inferir que a valores muy pequeños de la concentración inhibitoria del $50 \%\left(\mathrm{IC}_{50}\right),{ }^{72}$ el complejo posee una marcada reactividad, de modo que, se puede establecer un orden relativo de reactividad y contrastarlo con la reactividad predicha teóricamente en este trabajo

Así por ejemplo, se observa que la especie más reactiva (activa) corresponde a cisplatino, y el orden de reactividad en ambas líneas

Tabla 3. Parámetros geométricos calculados en fase gas y acuosa (mostrados en paréntesis) para los derivados I y VI. Longitudes de enlace en $\AA$ y ángulos de enlace en grados

\begin{tabular}{|c|c|c|c|}
\hline \multicolumn{4}{|c|}{ Funcionales } \\
\hline $\begin{array}{l}\text { Parámetro } \\
\text { geométrico }\end{array}$ & B3LYP & mPW1PW & HCTH \\
\hline \multicolumn{4}{|c|}{ Complejo I } \\
\hline$r(\mathrm{Pt}-\mathrm{Cl1})$ & $2,327(2,372)$ & $2,304(2,345)$ & $2,314(2,363)$ \\
\hline$r(\mathrm{Pt}-\mathrm{Cl} 2)$ & $2,301(2,324)$ & $2,277(2,299)$ & $2,286(2,313)$ \\
\hline$r(\mathrm{O} 1-\mathrm{C} 1)$ & $1,381(1,388)$ & $1,372(1,376)$ & $1,381(1,387)$ \\
\hline$r(\mathrm{C} 1-\mathrm{O} 2)$ & $1,198(1,212)$ & $1,194(1,193)$ & $1,200(1,199)$ \\
\hline$r(\mathrm{Pt}-\mathrm{O} 1)$ & $2,171(2,123)$ & $2,143(2,096)$ & $2,209(2,170)$ \\
\hline$r(\mathrm{Pt}-\mathrm{N} 1)$ & $2,108(2,081)$ & $2,078(2,055)$ & $2,099(2,075)$ \\
\hline$r(\mathrm{~N} 1-\mathrm{C} 2)$ & $1,496(1,501)$ & $1,484(1,489)$ & $1,485(1,488)$ \\
\hline$r(\mathrm{C} 1-\mathrm{C} 2)$ & $1,536(1,526)$ & $1,528(1,516)$ & $1,535(1,528)$ \\
\hline$\angle(\mathrm{Cl} 1-\mathrm{Pt}-\mathrm{Cl} 2)$ & $96,088(92,879)$ & $95,862(174,524)$ & $95,565(93,242)$ \\
\hline$\angle(\mathrm{O} 1-\mathrm{Pt}-\mathrm{N} 1)$ & $77,771(78,595)$ & $78,111(79,250)$ & $78,042(78,383)$ \\
\hline$\angle(\mathrm{Pt}-\mathrm{O} 1-\mathrm{C} 1)$ & $115,324(118,006)$ & $114,360(118,049)$ & $114,390(115,902)$ \\
\hline$\angle(\mathrm{Pt}-\mathrm{N} 1-\mathrm{C} 2)$ & $113,570(114,236)$ & $112,606(113,827)$ & $112,968(113,452)$ \\
\hline$\angle(\mathrm{Cl} 1-\mathrm{Pt}-\mathrm{N} 1)$ & $172,201(174,085)$ & $172,412(174,524)$ & $172,745(174,023)$ \\
\hline$\angle(\mathrm{Cl} 2-\mathrm{Pt}-\mathrm{O} 1)$ & $169,448(171,603)$ & $169,773(172,035)$ & $169,577(170,845)$ \\
\hline$\angle(\mathrm{O} 1-\mathrm{C} 1-\mathrm{C} 2)$ & $113,603(113,146)$ & $113,443(112,683)$ & $110,462(100,850)$ \\
\hline$\angle(\mathrm{N} 1-\mathrm{C} 2-\mathrm{C} 1)$ & $113,411(112,794)$ & $113,152(112,372)$ & $110,857(111,136)$ \\
\hline \multicolumn{4}{|c|}{ Complejo VI } \\
\hline$r(\mathrm{Pt}-\mathrm{O} 1)$ & $1,969(1,989)$ & $1,951(1,971)$ & $1,979(1,992)$ \\
\hline$r(\mathrm{Pt}-\mathrm{O} 2)$ & $1,987(2,026)$ & $1,971(2,009)$ & $1,995(2,032)$ \\
\hline$r(\mathrm{O} 3-\mathrm{C} 1)$ & $1,386(1,391)$ & $1,376(1,380)$ & $1,382(1,388)$ \\
\hline$r(\mathrm{C} 1-\mathrm{O} 4)$ & $1,197(1,196)$ & $1,193(1,193)$ & $1,200(1,199)$ \\
\hline$r(\mathrm{Pt}-\mathrm{O} 3)$ & $2,188(2,135)$ & $2,154(2,107)$ & $2,234(2,165)$ \\
\hline$r(\mathrm{Pt}-\mathrm{N} 1)$ & $2,113(2,085)$ & $2,084(2,059)$ & $2,210(2,082)$ \\
\hline$r(\mathrm{~N} 1-\mathrm{C} 2)$ & $1,496(1,499)$ & $1,485(1,488)$ & $1,489(1,491)$ \\
\hline$r(\mathrm{C} 1-\mathrm{C} 2)$ & $1,538(1,530)$ & $1,530(1,522)$ & $1,536(1,528)$ \\
\hline$\angle(\mathrm{O} 1-\mathrm{Pt}-\mathrm{O} 2)$ & $94,659(90,666)$ & $94,788(90,660)$ & $93,617(90,257)$ \\
\hline$\angle(\mathrm{O} 1-\mathrm{Pt}-\mathrm{N} 1)$ & $91,951(94,216)$ & $91,613(94,044)$ & $92,753(94,506)$ \\
\hline$\angle(\mathrm{O} 2-\mathrm{Pt}-\mathrm{O} 3)$ & $94,810(96,766)$ & $94,453(96,557)$ & $96,340(97,736)$ \\
\hline$\angle(\mathrm{O} 1-\mathrm{Pt}-\mathrm{O} 3)$ & $170,116(172,360)$ & $170,391(172,567)$ & $169,623(171,794)$ \\
\hline$\angle(\mathrm{O} 2-\mathrm{Pt}-\mathrm{N} 1)$ & $172,165(174,571)$ & $172,372(174,701)$ & $172,348(174,687)$ \\
\hline$\angle(\mathrm{O} 3-\mathrm{Pt}-\mathrm{N} 1)$ & $78,401(78,285)$ & $78,982(78,669)$ & $77,105(77,432)$ \\
\hline$\angle(\mathrm{O} 3-\mathrm{C} 1-\mathrm{C} 2)$ & $110,887(110,796)$ & $110,839(110,770)$ & $110,365(110,376)$ \\
\hline$\angle(\mathrm{N} 1-\mathrm{C} 2-\mathrm{C} 1)$ & $109,318(109,090)$ & $109,415(109,239)$ & $109,091(109,213)$ \\
\hline
\end{tabular}


Tabla 4. Descriptores globales y locales calculados al nivel mPW1PW/LANL2DZ*

\begin{tabular}{|c|c|c|c|c|c|c|c|c|c|c|c|}
\hline \multicolumn{12}{|c|}{ FASE GAS } \\
\hline Complejo & $\mathrm{E}_{\text {номо }}(\mathrm{eV})$ & $\mathrm{E}_{\text {LUMO }}(\mathrm{eV})$ & $\mu(\mathrm{eV})$ & $\eta(\mathrm{eV})$ & $W(\mathrm{eV})$ & $\mathrm{S}(\mathrm{eV})$ & $f_{k}^{+}(\mathrm{eV})$ & $\omega^{+}(\mathrm{eV})$ & $s^{+}{ }_{k}(\mathrm{eV})$ & $(s f)_{k}^{+}(\mathrm{eV})$ & $(\omega f)_{k}^{+}((\mathrm{eV})$ \\
\hline 1 & $-6,42$ & $-1,25$ & $-3,837$ & 2,585 & 2,847 & 0,193 & $-0,242$ & $-0,689$ & $-0,047$ & 0,011 & 0,167 \\
\hline 2 & $-6,31$ & $-0,63$ & $-3,469$ & 2,844 & 2,117 & 0,176 & $-0,054$ & $-0,115$ & $-0,010$ & 0,001 & 0,006 \\
\hline 3 & $-6,23$ & $-1,33$ & $-3,782$ & 2,449 & 2,921 & 0,204 & $-0,157$ & $-0,457$ & $-0,032$ & 0,005 & 0,072 \\
\hline 4 & $-6,31$ & $-1,03$ & $-3,674$ & 2,640 & 2,556 & 0,189 & $-0,177$ & $-0,451$ & $-0,033$ & 0,006 & 0,080 \\
\hline 5 & $-6,07$ & $-1,03$ & $-3,551$ & 2,517 & 2,505 & 0,199 & $-0,136$ & $-0,340$ & $-0,027$ & 0,004 & 0,046 \\
\hline 6 & $-6,15$ & $-0,87$ & $-3,51$ & 2,640 & 2,334 & 0,189 & $-0,161$ & $-0,375$ & $-0,030$ & 0,005 & 0,060 \\
\hline 7 & $-6,04$ & $-1,06$ & $-3,551$ & 2,490 & 2,532 & 0,201 & $-0,104$ & $-0,262$ & $-0,021$ & 0,002 & 0,027 \\
\hline 8 & $-6,15$ & $-0,90$ & $-3,524$ & 2,626 & 2,364 & 0,190 & $-0,113$ & $-0,268$ & $-0,022$ & 0,002 & 0,030 \\
\hline 9 & $-6,50$ & $-2,07$ & $-4,286$ & 2,218 & 4,141 & 0,225 & $-0,217$ & $-0,900$ & $-0,049$ & 0,011 & 0,196 \\
\hline 10 & $-6,56$ & $-2,18$ & $-4,367$ & 2,191 & 4,354 & 0,228 & $-0,198$ & $-0,863$ & $-0,045$ & 0,009 & 0,171 \\
\hline \multicolumn{12}{|c|}{ FASE ACUOSA } \\
\hline Complejo & $\mathrm{E}_{\text {Номо }}(\mathrm{eV})$ & $\mathrm{E}_{\text {LUMO }}(\mathrm{eV})$ & $\mu(\mathrm{eV})$ & $\eta(\mathrm{eV})$ & $W(\mathrm{eV})$ & $\mathrm{S}(\mathrm{eV})$ & $f_{k}^{+}(\mathrm{eV})$ & $\omega^{+}{ }_{k}(\mathrm{eV})$ & $s^{+}{ }_{k}(\mathrm{eV})$ & $(s f)_{k}^{+}(\mathrm{eV})$ & $(\omega f)_{k}^{+}((\mathrm{eV})$ \\
\hline 1 & $-6,993$ & $-1,361$ & $-4,177$ & 2,816 & 3,097 & 0,355 & $-0,305$ & $-0,944$ & $-0,108$ & 0,033 & 0,288 \\
\hline 2 & $-6,803$ & $-0,408$ & $-3,606$ & 3,197 & 2,033 & 0,313 & $-0,311$ & $-0,633$ & $-0,097$ & 0,030 & 0,197 \\
\hline 3 & $-6,993$ & $-1,442$ & $-4,218$ & 2,776 & 3,205 & 0,360 & $-0,288$ & $-0,923$ & $-0,104$ & 0,030 & 0,266 \\
\hline 4 & $-7,021$ & $-1,333$ & $-4,177$ & 2,844 & 3,068 & 0,352 & $-0,283$ & $-0,869$ & $-0,100$ & 0,028 & 0,246 \\
\hline 5 & $-6,884$ & $-1,361$ & $-4,123$ & 2,762 & 3,077 & 0,362 & $-0,279$ & $-0,846$ & $-0,101$ & 0,028 & 0,240 \\
\hline 6 & $-6,939$ & $-1,279$ & $-4,109$ & 2,830 & 2,983 & 0,353 & $-0,282$ & $-0,854$ & $-0,100$ & 0,028 & 0,237 \\
\hline 7 & $-6,912$ & $-1,361$ & $-4,136$ & 2,776 & 3,082 & 0,360 & $-0,286$ & $-0,895$ & $-0,103$ & 0,030 & 0,253 \\
\hline 8 & $-6,939$ & $-1,279$ & $-4,109$ & 2,830 & 2,983 & 0,353 & $-0,283$ & $-0,856$ & $-0,100$ & 0,028 & 0,239 \\
\hline 9 & $-6,884$ & $-2,259$ & $-4,572$ & 2,313 & 4,518 & 0,432 & $-0,314$ & $-1,418$ & $-0,136$ & 0,043 & 0,445 \\
\hline 10 & $-6,776$ & $-2,367$ & $-4,572$ & 2,204 & 4,741 & 0,454 & $-0,310$ & $-1,402$ & $-0,141$ & 0,044 & 0,457 \\
\hline
\end{tabular}

de células viene dado como; $1>9>6>2>10>5>3>4>7>8$ para HeLa y $1>2>9>5>4>10>6>3>8>7$ para HL-60, respectivamente. Para establecer el orden de reactividad de la serie de electrófilos estudiados (complejos de cis-platino(II)), se tuvo en cuenta el índice de electrofilicidad global, de tal manera, que el orden de reactividad en la fase gas es $10>9>3>1>4>7>5>8>6>2$ y acuosa $10>9>3>7>1>5>4>6=8>2$ respectivamente. Aunque, los órdenes de reactividad experimentales no coinciden completamente con los teóricos, es claro que los ligandos portadores influyen notablemente sobre la reactividad de los complejos de cis-platino(II). Asimismo, los valores de $W$ predichos para cisplatino, mostraron la mayor reactividad de este complejo en relación a carboplatino, lo cual está en total acuerdo con lo observado experimentalmente. Por otro lado, de acuerdo con el principio de máxima dureza de Pearson, ${ }^{50}$ la estructura más estable fue carboplatino, ya que se obtuvo el mínimo valor de índice de electrofilicidad y máximo potencial químico electrónico. Los complejos D y L-ornitina, presentaron los valores más altos de $W$, sugiriendo que la coordinación directa de ligandos portadores aminoácidos al centro metálico, podrían incrementar la reactividad del complejo.

Ahora bien, el hecho que estos derivados incrementen su reactividad en relación a cisplatino, no implica que su acción citostática sea superior (Tabla 5), ya que, se deben considerar otros factores que interfieran en la actividad del fármaco. Así por ejemplo, en la Tabla $5, \mathrm{PtCl}_{2}$ (dap-phe) y $\mathrm{PtCl}_{2}$ (dab-phe) muestran una actividad citotóxica significativamente baja, aunque tengan unido un ligando aminoácido. Moradell y colaboradores, ${ }^{24}$ sugirieron, que esta disminución en la actividad de $\mathrm{PtCl}_{2}$ (dap-phe) y $\mathrm{PtCl}_{2}$ (dab-phe), se debía a la cadena lateral voluminosa de fenilalanina, la cual hace difícil el paso través de la membrana celular, ${ }^{24}$ denotando por tanto, un factor limitante como lo es la solubilidad a través de la membrana celular. Se puede afirmar entonces, que la reactividad de los complejos derivados de cisplatino donde se coordina un ligando aminoácido aumenta su reactividad, pero en condiciones fisiológicas se debe tener en cuenta que una cadena lateral voluminosa no presenta un efecto positivo sobre la citotoxicidad del potencial fármaco. Los altos valores de $W$ para los complejos D y L-ornitina, en comparación con cisplatino, sugieren que la menor actividad citotóxica de estos fármacos puede estar relacionada con la ocurrencia de interacciones tipo electrófilo/ nucleófilo con otras biomoléculas antes de alcanzar el ADN, ya que al ser más reactivas su selectividad disminuye y pueden ser atacados por otras moléculas donadoras de electrones disponibles en el medio intracelular. Esta idea se puede soportar, considerando que los principales mecanismos de resistencia observados para cisplatino se deben principalmente a interacciones no específicas con otras macromoléculas, además del ADN y que generalmente contienen azufre, destacándose glutatión y metalotioneínas. ${ }^{73}$

Tabla 5. Valores experimentales de $\mathrm{IC}_{50}$ en $\mu \mathrm{M}$ para los complejos estudiados

\begin{tabular}{lcc}
\hline Complejos & \multicolumn{2}{c}{ Líneas celulares cancerosas } \\
\cline { 2 - 3 } & $\mathrm{HeLa}$ & $\mathrm{HL}-60$ \\
\hline cisplatino (1) & $2,5^{\mathrm{a}}$ & $2,5^{\mathrm{a}}$ \\
carboplatino (2) & $65^{\mathrm{a}}$ & $30^{\mathrm{a}}$ \\
$\mathrm{PtCl}_{2}$ (dap) (3) & $165^{\mathrm{b}}$ & $160^{\mathrm{b}}$ \\
$\mathrm{PtCl}_{2}$ (dab) (4) & $215^{\mathrm{b}}$ & $95^{\mathrm{b}}$ \\
$\mathrm{PtCl}_{2}$ (dap-ala) (5) & $160^{\mathrm{a}}$ & $90^{\mathrm{a}}$ \\
$\mathrm{PtCl}_{2}$ (dab-ala) (6) & $60^{\mathrm{a}}$ & $130^{\mathrm{a}}$ \\
$\mathrm{PtCl}_{2}$ (dap-phe) (7) & $335^{\mathrm{a}}$ & $340^{\mathrm{a}}$ \\
$\mathrm{PtCl}_{2}$ (dab-phe) (8) & $440^{\mathrm{a}}$ & $330^{\mathrm{a}}$ \\
$\mathrm{PtCl}_{2}[\mathrm{D}-(\mathrm{N}, \mathrm{O})$-ornitina $](9)$ & $50 \pm 4^{\mathrm{c}}$ & $70 \pm 6^{\mathrm{c}}$ \\
$\mathrm{PtCl}_{2}[\mathrm{~L}-(\mathrm{N}, \mathrm{O})$-ornitina $](10)$ & $125 \pm 7^{\mathrm{c}}$ & $123 \pm 9^{\mathrm{c}}$ \\
\hline
\end{tabular}

${ }^{\mathrm{a} R e f} 24$; ${ }^{\mathrm{b}} \operatorname{Ref} 25$; $\mathrm{c} \operatorname{Ref} 10$.

No obstante, la evidencia experimental ${ }^{10}$ sugiere que los complejos D y L-ornitina, son sustancialmente selectivos, ya que el 
Tabla 6. Descriptores globales y locales calculados al nivel mPW1PW/LANL2DZ* para los derivados propuestos

\begin{tabular}{|c|c|c|c|c|c|c|c|c|c|c|c|}
\hline \multicolumn{12}{|c|}{ FASE GAS } \\
\hline Complejo & $\mathrm{E}_{\text {номо }}(\mathrm{eV})$ & $\mathrm{E}_{\text {LUMO }}(\mathrm{eV})$ & $\mu(\mathrm{eV})$ & $\eta(\mathrm{eV})$ & $W(\mathrm{eV})$ & $\mathrm{S}(\mathrm{eV})$ & $f^{+}{ }_{k}(\mathrm{eV})$ & $\omega^{+}(\mathrm{eV})$ & $s_{k}^{+}(\mathrm{eV})$ & $(s f)_{k}^{+}(\mathrm{eV})$ & $(\omega f)_{k}^{+}((\mathrm{eV})$ \\
\hline I & $-6,531$ & $-2,095$ & $-4,31$ & 2,22 & 4,19 & 0,45 & $-0,216$ & $-0,906$ & $-0,097$ & 0,021 & 0,196 \\
\hline II & $-6,367$ & $-1,850$ & $-4,11$ & 2,26 & 3,74 & 0,44 & $-0,113$ & $-0,422$ & $-0,050$ & 0,006 & 0,048 \\
\hline III & $-6,395$ & $-1,959$ & $-4,18$ & 2,22 & 3,93 & 0,45 & $-0,186$ & $-0,733$ & $-0,084$ & 0,016 & 0,136 \\
\hline IV & $-6,259$ & $-1,742$ & $-4,00$ & 2,26 & 3,54 & 0,44 & $-0,085$ & $-0,301$ & $-0,038$ & 0,003 & 0,026 \\
\hline V & $-6,531$ & $-2,068$ & $-4,30$ & 2,23 & 4,14 & 0,45 & $-0,207$ & $-0,856$ & $-0,093$ & 0,019 & 0,177 \\
\hline VI & $-6,367$ & $-1,850$ & $-4,11$ & 2,26 & 3,74 & 0,44 & $-0,121$ & $-0,452$ & $-0,054$ & 0,006 & 0,055 \\
\hline VII & $-6,558$ & $-2,204$ & $-4,38$ & 2,18 & 4,41 & 0,46 & $-0,189$ & $-0,833$ & $-0,087$ & 0,016 & 0,157 \\
\hline VIII & $-6,395$ & $-1,959$ & $-4,18$ & 2,22 & 3,93 & 0,45 & $-0,120$ & $-0,473$ & $-0,054$ & 0,007 & 0,057 \\
\hline IX & $-6,422$ & $-1,986$ & $-4,20$ & 2,22 & 3,98 & 0,45 & $-0,203$ & $-0,809$ & $-0,091$ & 0,019 & 0,164 \\
\hline $\mathrm{X}$ & $-6,259$ & $-1,714$ & $-3,99$ & 2,27 & 3,50 & 0,44 & $-0,103$ & $-0,362$ & $-0,046$ & 0,005 & 0,037 \\
\hline \multicolumn{12}{|c|}{ FASE ACUOSA } \\
\hline I & $-7,184$ & $-2,231$ & $-4,71$ & 2,48 & 4,47 & 0,40 & $-0,303$ & $-1,357$ & $-0,122$ & 0,037 & 0,411 \\
\hline II & $-7,021$ & $-1,388$ & $-4,20$ & 2,82 & 3,14 & 0,36 & $-0,320$ & $-1,005$ & $-0,114$ & 0,036 & 0,322 \\
\hline III & $-5,932$ & $-2,259$ & $-4,10$ & 1,84 & 4,57 & 0,54 & $-0,312$ & $-1,426$ & $-0,170$ & 0,053 & 0,445 \\
\hline IV & $-5,905$ & $-1,388$ & $-3,65$ & 2,26 & 2,94 & 0,44 & $-0,313$ & $-0,920$ & $-0,138$ & 0,043 & 0,288 \\
\hline V & $-7,184$ & $-2,231$ & $-4,71$ & 2,48 & 4,47 & 0,40 & $-0,312$ & $-1,395$ & $-0,126$ & 0,039 & 0,435 \\
\hline VI & $-7,021$ & $-1,388$ & $-4,20$ & 2,82 & 3,14 & 0,36 & $-0,314$ & $-0,985$ & $-0,111$ & 0,035 & 0,309 \\
\hline VII & $-6,340$ & $-2,286$ & $-4,31$ & 2,03 & 4,59 & 0,49 & $-0,308$ & $-1,413$ & $-0,152$ & 0,047 & 0,435 \\
\hline VIII & $-6,531$ & $-2,259$ & $-4,39$ & 2,14 & 4,52 & 0,47 & $-0,285$ & $-1,290$ & $-0,134$ & 0,038 & 0,368 \\
\hline IX & $-6,531$ & $-2,259$ & $-4,39$ & 2,14 & 4,52 & 0,47 & $-0,310$ & $-1,399$ & $-0,145$ & 0,045 & 0,433 \\
\hline $\mathrm{X}$ & $-6,531$ & $-1,415$ & $-3,97$ & 2,56 & 3,09 & 0,39 & $-0,259$ & $-0,800$ & $-0,101$ & 0,026 & 0,208 \\
\hline
\end{tabular}

aminoácido ornitina, es uno de los precursores en la biosíntesis natural de poliaminas y policationes, los cuales son altamente requeridos por células tumorales, y en consecuencia, el transporte de estos complejos hacia el interior de la célula es más eficientemente. ${ }^{10}$ Esto último, soportaría el hecho que los complejos D y L-ornitina, presentan mayor actividad que carboplatino y los complejos $\mathrm{PtCl}_{2}$ (dap-ala), $\mathrm{PtCl}_{2}$ (dab-ala), $\mathrm{PtCl}_{2}$ (dap-phe) y $\mathrm{PtCl}_{2}$ (dab-phe) respectivamente, para la línea celular cancerosa HeLa. De lo anterior es claro que, el uso de ligandos portadores (LP), fisiológicamente activos (aminoácidos), confiere propiedades interesantes al complejo derivado y por ende el diseño de nuevos análogos conteniendo este tipo de ligandos debe ser considerado. En este orden de ideas, se estudio la reactividad de 10 análogos de cis-platino (II) (Figura 1b), que contienen en su estructura ligandos portadores aminoácidos. La reactividad predicha para estos complejos (Tabla 6), muestra una tendencia similar a la calculada para los complejos D y L-ornitina. Tanto en la fase gas como en la acuosa, los complejos con ligandos salientes cloruro fueron más reactivos que aquellos con ligandos salientes del tipo, 1,1'-ciclobutanodicarboxilato, lo cual es cualitativamente consistente con las diferencias de reactividad encontradas entre cisplatino y carboplatino a nivel experimental (Tabla 5), donde la sustitución de los ligandos cloruro en cisplatino por el grupo 1,1'-ciclobutanodicarboxilato en carboplatino, disminuye notablemente la reactividad de este último. ${ }^{22}$ Esta disminución de la reactividad no siempre representa una desventaja para el complejo anticanceroso derivado, puesto que, el ligando 1,1'-ciclobutanodicarboxilato disminuye significativamente los efectos colaterales inducidos por cisplatino y sin que se afecte la actividad antitumoral del mismo. ${ }^{74}$ Por lo tanto, se podría esperar un comportamiento reactivo similar para los complejos II, IV, VI, VIII y $\mathrm{X}$ respectivamente, ya que estos presentan este ligando saliente. Ahora bien, es interesante notar que la reactividad de todos los complejos propuestos que tienen unido el grupo 1,1'-ciclobutanodicarboxilato, es más alta que la obtenida para carboplatino, sugiriendo con ello que la unión de los aminoácidos mejora las características reactivas del complejo derivado. Por lo tanto, a la luz de la reactividad experimental observada para carboplatino y los complejos D y L-ornitina, así como también, los cálculos teóricos, se presume que los complejos II, IV, VI, VIII y X tengan potencialidad anticancerosa y conserven las características de baja reactividad y alta selectividad de sus análogos sintetizados (D y L-ornitina). Lo anterior, constituye un objetivo deseable dentro de las nuevas estrategias para el diseño racional de fármacos anticancerosos basados en cis-platino(II), ya que, se busca sintetizar derivados que sean seguros para las células sanas, con elevada actividad antitumoral, selectividad y que presenten pocos o nulos efectos colaterales. ${ }^{12,14}$ Por otro lado, cabe resaltar que el complejo VIII, mostró una reactividad significativa en fase acuosa en relación al resto de complejos con el ligando saliente 1,1 '-ciclobutanodicarboxilato, indicando que el LP tirosina favorece la reactividad del derivado. En las Tablas 4 y 6, los valores altos de blandura, confirman que los complejos más reactivos son aquellos que modifican fácilmente su densidad electrónica.

Con respecto a los descriptores locales, FF $\left(f_{k}^{+}\right)$, la filicidad $\left(\omega_{k}^{+}\right)$ y la suavidad local $\left(s_{k}^{+}\right)$, respectivamente, se presentan los valores calculados para el átomo de platino solamente, en fase gas y acuosa dado que el mecanismo de unión de $\mathrm{ADN}$ a los fármacos anticancerosos basados en platino, implica el ataque nucleofílico sobre dicho átomo. Para el resto de átomos, estas cantidades no son reportadas aquí, pero se trataran más adelante dentro del contexto de los descriptores de grupo. De las Tablas 4 y 6, en ambas fases se observa la ocurrencia de valores negativos para FF. De acuerdo con Roy y colaboradores, ${ }^{75}$ estos valores negativos se deben a que en la definición analítica de FF, dicha función es válida solamente cuando $\Delta \mathrm{N} \rightarrow 0$. Para tal caso, la fluctuación en la distribución de la densidad electrónica es mínima, ya que la respectiva relajación en la especie catiónica o aniónica es despreciable. ${ }^{75}$ Sin embargo, en la definición de $f_{k}^{\alpha}$ condensada (calculada por el método de diferencias finitas), se permite que el número de electrones cambie en una cantidad mayor que uno y como resultado se da un cambio significativo en la distribución de la densidad electrónica, principalmente debido a una relajación significativa en las especies catiónicas y aniónicas. Lo anterior, trae consigo la 
acumulación o disminución de la densidad electrónica sobre un sitio particular de la molécula, aun cuando el número de electrones aumente o disminuya en uno.$^{75}$ Por otro lado, Ayres, ${ }^{43}$ recientemente relacionó el comportamiento anteriormente expuesto con la reducción u oxidación de centros atómicos en determinada molécula. Estos valores negativos constituyen un verdadero rompecabezas a la hora de analizar los datos, así que, dentro de las estrategias para superar la complejidad asociada a dichos valores negativos para FF, Kolandaivel y colaboradores, ${ }^{32}$ propusieron el descriptor atómico $(s f)_{k}^{\alpha}$, con el cual obtuvieron resultados confiables para las tendencias de reactividad de un grupo de moléculas seleccionadas. Además, como se indicó previamente, en este trabajo se introdujo el nuevo descriptor atómico de filicidad $(\omega f)_{k}^{\alpha}$, para analizar la reactividad y selectividad de los diferentes sitios (átomos) de los complejos estudiados. Así, la descripción de la reactividad para el grupo de moléculas estudiadas, desde el punto de vista local, se llevó a cabo teniendo en cuenta los descriptores atómicos, dado que ambos contienen información tanto de descriptores locales como globales y evitan los valores negativos para FF. A este propósito, en las Tablas 4 y 6 se presentan los valores calculados para los descriptores atómicos arriba mencionados, indicando que los átomos de platino más electrofílicos en los diferentes complejos son aquellos donde se obtuvieron los máximos valores de $(s f)_{k}^{+}, \mathrm{y}(\omega f)_{k}^{+}$respectivamente, los cuales a su vez coinciden con las regiones más blandas en la molécula, es decir, donde la densidad electrónica se modifica más fácilmente. Así por ejemplo, de la Tabla 4 se observa que en el cambio de fase gas a fase acuosa, la reactividad para todos los complejos aumenta, indicando con ello que los átomos de platino en todos los complejos son químicamente más blandos. Asimismo, carboplatino, presenta el átomo de platino menos reactivo de la serie, lo cual es consecuente con la baja reactividad de este complejo, ya que, su comportamiento como electrófilo durante la reacción con $\mathrm{ADN}$ está íntimamente relacionado con su comportamiento local. Con respecto al nuevo descriptor $(\omega f)_{k}^{+}$, es evidente que reproduce las tendencias de reactividad local esperada para cada complejo (Tabla 4). Este resultado no sorprende, si se tiene en cuenta que el descriptor $(\omega f)_{k}^{+}$, reúne varios descriptores globales y locales de la reactividad, así como también, de selectividad y por ende ofrece información confiable sobre el poder nucleofílico o electrofílico en diferentes sitios atómicos de las moléculas investigadas.

Para los complejos propuestos (Tabla 6), los descriptores, $(s f)_{k}^{+}$y $(\omega f)_{k}^{+}$, muestran tendencias de reactividad similares a las predichas para los complejos D y L-ornitina en fase gas, sin embargo, en la fase acuosa la reactividad del átomo de platino en los diferentes complejos es sutilmente menor. No obstante, los complejos II, IV, VI, VIII y X, mostraron valores de $(s f)_{k}^{+}$y $(\omega f)_{k}^{+}$, sustancialmente altos en relación a carboplatino; especialmente el complejo VIII, para el cual en la fase acuosa se obtuvo el máximo valor de $(\omega f)_{k}^{+}$. Este resultado, indica la capacidad del átomo de platino en VIII, para aceptar densidad electrónica. Por otro lado, de la Tabla 6, también se observa que todos los complejos presentan energías LUMO muy negativas implicando con ello afinidades electrónicas altas y por lo tanto pueden acomodar fácilmente el exceso de electrones provenientes de los alrededores (ADN), lo que está en buen acuerdo con lo predicho por los descriptores $(s f)_{k}^{+}$y $(\omega f)_{k}^{+}$.

Para analizar los efectos del ambiente químico alrededor del átomo de platino en cada complejo, se calcularon los descriptores de grupo $(s f)_{g}^{\alpha}$ y $(\omega f)_{g}^{\alpha}$ de los átomos vecinos al centro metálico, (ligandos portadores; $\mathrm{LP}=\mathrm{N}, \mathrm{C}, \mathrm{O}, \mathrm{H}$ y ligando saliente; $\mathrm{LS}=$ 1,1'-ciclobutanodicarboxilato), mediante las Ecuaciones $16 \mathrm{a}$ y $16 \mathrm{~b}$ respectivamente.

Ahora bien, con el fin de explorar la capacidad predictiva de los descriptores de grupo y la contribución de los LP y LS a la reactividad del átomo de platino, los valores predichos para $(s f)_{g}^{\alpha} \mathrm{y}$ $(\omega f)_{g}^{\alpha}$, (todos los átomos de LP y LS respectivamente) se sumaron a los descriptores atómicos $(s f)_{k}^{+}$y $(\omega f)_{k}^{+}$calculados para el átomo de platino en cada complejo (Tablas $1 \mathrm{~S}$ y $2 \mathrm{~S}$, material suplementario). En las Tablas $1 \mathrm{~S}$ y $2 \mathrm{~S}$, se presentan los valores de DG calculados en fase gas y acuosa respectivamente. En la Tabla $1 \mathrm{~S}$, se observa como los diferentes LP alrededor de platino modulan la reactividad del centro metálico. Así por ejemplo, en la fase gas, para el complejo cisplatino hay una contribución significativa de su LP a la reactividad del átomo platino al compararlo con el resto de complejos. Asimismo, para los complejos D y L-ornitina en fase gas, la contribución del LP aminoácido, también es significativa si se compara con otros ligandos portadores. Otro resultado interesante, que se observa en la Tabla 1S, es que los complejos que tienen LP aminoácidos con cadenas laterales voluminosas ( 7 y 8), no contribuyen de manera significativa al incremento del poder de aceptación de densidad electrónica del átomo de platino. Este comportamiento es consecuente con lo esperado, pues, la reactividad de estas moléculas es muy baja en comparación con los el resto de complejos presentado en la Tabla 5. Para los complejos D y L-ornitina se observa una contribución apreciable del LP ornitina sobre la reactividad del centro metálico, por lo que es factible sugerir que la presencia de estos grupos en el complejo derivado efectivamente aumentan la reactividad de Pt.

Para los complejos propuestos (Tabla 2S), cabe resaltar que la combinación de LP aminoácido con LS del tipo 1,1'-ciclobutanodicarboxilato, aumenta sustancialmente el poder de aceptación de densidad electrónica del centro metálico, por lo que a nivel local se esperaría marcada reactividad y selectividad del átomo de platino frente a la molécula de ADN.

Para el derivado VIII, se observó, que los ligandos portador y saliente aumentan la reactividad de Pt y por ende la selectividad sobre este sitio frente al ataque nucleofílico. Por otro lado, como se esperaba las moléculas con grupo saliente cloruro (Tabla $2 \mathrm{~S}$ ), fueron más reactivas que su contraparte con 1,1'-ciclobutanodicarboxilato. De lo anterior es claro que, los nuevos descriptores de grupo basados en el descriptor atómico de Kolandaivel y colaboradores ${ }^{32}$ y el descriptor atómico de filicidad $(\omega f)_{k}^{+}$, permiten obtener resultados confiables en la predicción cualitativa de la reactividad local del grupo moléculas estudiadas. Por lo tanto, el uso de la información estructural y de reactividad arrojada por estos descriptores como base para la síntesis de nuevos complejos derivados de cisplatino, constituye una pieza clave en la búsqueda de estrategias orientadas hacia el diseño racional de nuevos agentes anticancerosos con propiedades fisicoquímicas y farmacológicas superiores a los medicamentos de uso actual en el tratamiento de este tipo de patologías.

\section{CONCLUSIONES}

De los resultados expuestos en este trabajo, pueden extraerse varias conclusiones entre las cuales cabe destacar que el funcional mPW1PW en combinación con el pseudopotencial LANL2DZ aumentado con funciones de polarización d (LANL2DZ*), permitieron localizar geometrías de equilibrio en buen acuerdo con las geometrías experimentales. La inclusión del solvente en todos los casos mejoró notablemente la descripción de las geometrías de equilibrio predichas mediante el protocolo $\mathrm{mPW} 1 \mathrm{PW} / \mathrm{LANL} 2 \mathrm{DZ} *$.

Por otro lado, es evidente que los descriptores globales de la reactividad basados en la DFT, fueron de gran utilidad para predecir de manera cualitativa, la estabilidad intrínseca, reactividad química y estabilidad relativa de los compuestos de cis-platino(II) estudiados.

El análisis de la reactividad local en términos de los descriptores atómicos, permitió obtener información profunda sobre la reactividad y selectividad de sitio de los diferentes átomos en las moléculas estudiadas; específicamente sobre el átomo de platino. Así 
por ejemplo, con el descriptor atómico de filicidad, se pudo inferir sobre el comportamiento local del átomo de platino frente a un ataque nucleofílico, puesto que dicho descriptor combina dos descriptores locales como lo son; la filicidad y la FF respectivamente, los cuales están estrechamente relacionados con la selectividad y reactividad de sitios atómicos en una molécula. Además, contiene información del descriptor global $W$. De modo que, sin ambigüedad se pudo establecer que los ligandos aminoácidos pueden modular el comportamiento electrofílico del átomo de platino.

La variación de la densidad electrónica alrededor del átomo de platino (sitio reactivo), debida a los cambios en la naturaleza de los ligandos portadores y salientes, es efectivamente reproducida por los descriptores de grupo, definidos en este trabajo, dando luces de la importancia que tiene este grupo de átomos sobre las características reactivas y biológicas de los fármacos anticancerosos de cis-platino(II).

\section{MATERIAL SUPLEMENTARIO}

Disponible en http://quimicanova.sbq.org.br, em forma de archivo PDF y com acesso libre.

\section{AGRADECIMIENTOS}

Los autores de este artículo muestran su agradecimiento al Centro de Investigaciones Universitarias (CIUC), de la Universidad de Córdoba, por el apoyo económico brindado (proyecto: código 1.2.08.109 y numeral FCB-03-09).

\section{REFERENCIAS}

1. Klein, C. D.; Bachelier, A.; J. Comput. Aided Mol. Des. 2006, 20, 621.

2. Roumen, L.; Sanders, M. P.; Pieterse, K.; Hilbers, P. A.; Plate, R.; Custers, E.; Gooyer, M.; Smits, J. F.; Beugels, I.; Emmen, J.; Ottenheijm, H. C.; Leysen, D.; Hermans, J. J.; J. Comput. Aided Mol Des. 2007, 21, 455 .

3. Hussain, A.; Melville, J. L.; Hirst, J. D.; J. Comput. Aided Mol. Des. 2010, 24,1

4. Bultinck, P.; Computational Medicinal Chemistry for Drug Discovery; De Winter, H.; Langenaeker, W.; Tollenaere, J.P.; Eds.; Marcel Dekker Inc: New York, 2003.

5. Carloni, P.; Alber, F.; Quantum Medicinal Chemistry; Mannhold, R; Kubinyi, H; Folkers, G., Eds.; Wiley-VCH Verlag GmbH \& Co, KGaA: Weinheim, 2005.

6. Rosenberg, B.; Vancamp, L.; Trosko, J. E.; Mansour, V. H.; Nature 1969, 222,385 .

7. Whiteside, M. A.; Piyathilake, C. J.; Bushell, T. M.; Johanning, G. L.; Nutr. Cancer 2006, 54, 274

8. Jamieson, E. R.; Lippard, S. J.; Chem. Rev. 1999, 99, 2467.

9. Komeda, S.; Lutz, M.; Spek, A. L.; Chikuma, M.; Reedijk, J.; Inorg. Chem. 2000, 39, 4230.

10. Dalla Via, L.; Gia, O.; Magno, S. M.; Dolmella, A.; Marton, D.; Di Noto, V.; Inorg. Chim. Acta 2006, 359, 4197.

11. Wu, C.; Wangpaichitr, M.; Feun, L.; Kuo, M.; Robles, C.; Lampidis, T.; Savaraj, N.; Mol. Cancer 2005, 4, 25.

12. Ye, Q. S.; Xie, M. J.; Liu, W. P.; Chen, X. Z.; Yu, Y.; Chang, Q. W.; Hou, S. Q.; Chem. Pharm. Bull. 2009, 57, 424.

13. Galanski, M.; Keppler, B. K.; Anticancer Agents Med. Chem. 2007, 7, 55.

14. Abu-Surrah, A. S.; Kettunen, M.; Curr. Med. Chem. 2006, 13, 1337.

15. Burke, K.; J. Chem. Phys. 2012, 136, 150901.

16. Moens, J.; Geerlings, P.; Roos, G.; Chem. Eur. J. 2007, 13, 8174.

17. De Vleeschouwer, F.; Van Speybroeck, V.; Waroquier, M.; Geerlings, P.; De Proft, F.; Org. Lett. 2007, 9, 2721.

18. Chermette, H.; J. Comput. Chem. 1999, 20, 129.
19. Padmanabhan, J.; Parthasarathi, R.; Subramanian, V.; Chattaraj, P. K.; Chem. Res. Toxicol. 2006, 19, 356.

20. Chattaraj, P. K.; Maiti, B.; Sarkar, U.; J. Phys. Chem. A 2003, 107, 4973.

21. Padmanabhan, J.; Parthasarathi, R.; Subramanian, V.; Chattaraj, P. K.; Bioorg. Med. Chem. 2006, 14, 1021.

22. Sarmah, P.; Deka, R. C.; Inter. J. Quant. Chem. 2008, 108, 1400.

23. Sarmah, P.; Deka, R. C.; J. Comput. Aided Mol. Des. 2009, 23, 343.

24. Moradell, S.; Lorenzo, J.; Rovira, A.; Van Zutphen, S.; Avilés, F. X.; Moreno, V.; De Llorens, R.; Martinez, M. A.; Reedijk, J.; Llobet, A.; J. Inorg. Biochem. 2004, 98, 1933.

25. Moradell, S.; Lorenzo, J.; Rovira, A.; Robillard, M. S.; Avilés, F. X.; Moreno, V.; De Llorens, R.; Martínez, M. A.; Reedijk, J.; Llobet, A.; J. Inorg. Biochem. 2003, 96, 493.

26. Romerosa, A.; Bergamini, P.; Bertolasi, V.; Canella, A.; Cattabriga, M.; Gavioli, R.; Mañas, S.; Mantovani, N.; Pellacani, L.; Inorg. Chem. 2004, 43, 905.

27. van Zutphen, S.; Reedijk, J.; Coord. Chem. Rev. 2005, 249, 2845.

28. Cleare, M. J.; Hoeschele, J. D.; Platinum Metals Review 1973, 17, 2.

29. Connors, T. A.; Cleare, M. J.; Harrap, K. R.; Cancer Treat. Rep. 1979, 63, 1499.

30. Monti, E.; Gariboldi, M.; Maiocchi, A.; Marengo, E.; Cassino, C.; Gabano, E.; Osella, D.; J. Med. Chem. 2005, 48, 857.

31. Krishnamurty, S.; Pal, S.; J. Phys. Chem. A 2000, 104, 7639.

32. Kolandaivel, P.; Praveena, G.; Selvarengan, P.; J. Chem. Sci. 2005, 117, 591.

33. Chattaraj, P. K.; Giri, S.; Annu. Rep. Prog. Chem. Sect. C: Phys. Chem. 2009, 105, 13.

34. Méndez, F.; Gázquez, J. L.; J. Am. Chem. Soc. 1994, 116, 9298

35. Mendoza-Huizar, L.; Rios-Reyes, H.; J. Mex. Chem. Soc. 2011, 55, 142

36. Cárdenas, C.; Tiznado, W.; Ayers, P. W.; Fuentealba, P.; J. Phys. Chem. A 2011, 115, 2325.

37. Li, Y.; Evans, J.; J. Am. Chem. Soc. 1995, 117, 7756

38. Parr, R. G.; Density-Functional Theory of Atoms and Molecules; Oxford University Press; Clarendon Press: New York, 1994

39. Maynard, A. T.; Huang, M.; Rice, W. G.; Covell, D. G.; PNAS 1998, 95 , 11578.

40. Vivas-Reyes, R.; Núñez-Zarur, F.; Martínez, E.; Org. Electron. 2008, 9 , 625.

41. Broeckaert, L.; Moens, J.; Roos, G.; De Proft, F.; Geerlings, P.; J. Phys. Chem. A 2008, 112, 12164

42. Fievez, T.; Weckhuysen, B. M.; Geerlings, P.; De Proft, F.; J. Phys. Chem. C 2009, 113, 19905.

43. Ayers, P. W.; Morrison, R. C.; Roy, R. K.; J. Phys. Chem. 2002, 116 , 8731.

44. Yang, W.; Mortier, W.J.; J. Am. Chem. Soc. 1986, 108, 5708.

45. Cárdenas, C.; Rabi, N.; Ayers, P. W.; Morell, C.; Jaramillo, P.; Fuentealba, P.; J. Phys. Chem. A 2009, 113, 8660.

46. Martínez, J. I.; Moncada, J. L.; Larenas, J. M.; J. Mol. Model. 2010, 16, 1825 .

47. Kulkarni, B. S.; Tanwar, A.; Pal, S.; J. Chem. Sci. 2008, 119, 489.

48. Lackner, K. S.; Zweig, G.; Phys. Rev. D: Part. Fields 1987, 36, 1562.

49. Hohenberg, P.; Kohn, W.; Phys. Rev. 1964, 136, B864.

50. Pearson, R. G.; Acc. Chem. Res. 1993, 26, 250.

51. Moncada, J. L.; Morán, G. S.; Quim. Nova. 2008, 31, 1255.

52. Geerlings, P.; De Proft, F.; Langenaeker, W.; In Advances in Quantum Chemistry; Per-Olov Löwdin, Ed.; Acad. Press: San Diego, 1998; Vol. 33, pp. 303-328.

53. Parr, R. G.; Szentpály, L. V.; Liu, S.; J. Am. Chem. Soc. 1999, 121, 1922.

54. Parr, R.G.; Yang, W.; J. Am. Chem. Soc. 1984, 106, 4049.

55. Dronskowski, R.; J. Am. Chem. Soc. 1992, 114, 7230.

56. Galembeck, S. E.; Caramori, G. F.; Quim. Nova. 2003, 26, 957.

57. Singh, R. N.; Kumar, A.; Tiwari, R. K.; Rawat, P.; Baboo, V.; Verma, D.; Spectrochim. Acta, Part A 2012, 92, 295. 
58. Parthasarathi, R.; Padmanabhan, J.; Elango, M.; Subramanian, V.; Chattaraj, P. K.; Chem. Phys. Lett. 2004, 394, 225.

59. Frisch, M. J.; Gaussian 09 (Rev. B.01), Gaussian Inc. Wallingford CT, 2009.

60. Becke, A. D.; J. Chem. Phys. 1993, 98, 5648.

61. Adamo, C.; Barone, V.; J. Chem. Phys. 1998, 108, 664.

62. Hamprecht, F. A.; Cohen, A.J.; Tozer, D. J.; Handy, N. C.; J. Chem. Phys. 1998, 109, 6264.

63. Jensen, F.; Introduction to Computational Chemistry; $2^{\text {th }}$ ed., John Wiley \& Sons Ltd: Chinchester, 2006.

64. Hirshfeld, F. L.; Theor. Chim. Acta 1977, 44, 129.

65. Meneses, L.; Tiznado, W.; Contreras, R.; Fuentealba, P.; Chem. Phys. Lett. 2004, 383, 181.

66. Tomasi, J.; Mennucci, B.; Cammi, R.; Chem. Rev. 2005, 105, 2999.

67. Cossi, M.; Barone, V.; Cammi, R.; Tomasi, J.; Chem. Phys. Lett. 1996, $255,327$.
68. Hay, P. J.; Wadt, W. R.; J. Chem. Phys 1985, 82, 270

69. Lopes, J. F.; Rocha, W. R.; Dos Santos, H. F.; Almeida, W. B.; J. Braz. Chem. Soc. 2010, 21, 887.

70. Wysokiński, R.; Michalska, D.; J. Comput. Chem. 2001, 22, 901.

71. Milburn, G. H.; Truter, M. R.; J. Chem. Phys. A 1966, 1609.

72. Sebaugh, J. L.; Pharm. Stat. 2011, 10, 128.

73. Huska, D.; Fabrik, I.; Baloun, J.; Adam, V.; Masarik, M.; Hubalek, J.; Vasku, A.; Trnkova, L.; Horna, A.; Zeman, L.; Kizek, R.; Sensors 2009, 9, 1355 .

74. Ardizzoni, A.; Boni, L.; Tiseo, M.; Fossella, F. V.; Schiller, J. H.; Paesmans, M.; Radosavljevic, D.; Paccagnella, A.; Zatloukal, P.; Mazzanti, P.; Bisset, D.; Rosell, R.; J. Natl. Cancer. Inst. 2007, 99, 847.

75. Roy, R. K.; Pal, S.; Hirao, K.; J. Chem. Phys. 1999, 110, 8236. 